\title{
What do we know about titanized polypropylene meshes? An evidence-based review of the literature
}

\author{
F. Köckerling $\cdot$ C. Schug-Pass
}

Received: 18 February 2013/Accepted: 2 November 2013/Published online: 20 November 2013

(C) The Author(s) 2013. This article is published with open access at Springerlink.com

\begin{abstract}
Purpose Despite the vast selection of brands available, nearly all synthetic meshes for hernia surgery continue to use one or other of three basic materials: polypropylene, polyester and ePTFE. These are used in combination with each other or with a range of additional materials such as titanium, omega 3, monocryl, PVDF and hyaluronate. This systematic review of all experimental and clinical studies is aimed at investigating whether titanized meshes confer advantages over other synthetic meshes in hernia surgery. Materials and methods A search of the medical literature from 2002 to 2012, as indexed by Medline, was performed, using the PubMed search engine (http://www.pubmed.gov). The search terms were: hernia mesh, titanium coating, lightweight mesh, TiMesh, mesh complications. All papers were graded according to the Oxford hierarchy of evidence.

Results Patients operated on with the Lichtenstein technique performed using the lightweight titanium-coated mesh have a shorter convalescence than those with the heavy-weight mesh Prolene. For inguinal hernias operated on with the TAPP technique and using a lightweight titanium-coated mesh in comparison to a heavy-weight Prolene mesh, the early postoperative convalescence seems to improve. Titanized meshes do exhibit a negative effect on sperm motility 1 year after a TEP operation, but not after 3 years. The laparoscopic IPOM technique with a titaniumcoated polypropylene mesh was associated with less postoperative pain in the short term, lower analgesic
\end{abstract}

\footnotetext{
F. Köckerling $(\square) \cdot$ C. Schug-Pass

Department of Surgery and Center of Minimally Invasive

Surgery, Vivantes Hospital Spandau, Academic Teaching

Hospital of Charité University Medicine, Neue Bergstraße 6,

13585 Berlin, Germany

e-mail: ferdinand.koeckerling@vivantes.de
}

consumption and a quicker return to everyday activities compared with the Parietex composite mesh.

Conclusion In clinical studies, the titanium-coated polypropylene mesh shows in inguinal hernia repair certain benefits compared with the use of older heavy-weight meshes.

Keywords Titanized polypropylene meshes . TiMesh $\cdot$ Mesh biocompatibility $\cdot$ Mesh fixation

\section{Introduction}

Despite the vast selection of brands available, nearly all synthetic meshes for hernia surgery continue to use one or other of three basic materials: polypropylene, polyester and ePTFE. These are used in combination with each other or with a range of additional materials such as titanium, omega 3, monocryl, PVDF and hyaluronate [1]. The ideal synthetic mesh should assure optimum biocompatibility, be easy to handle and provide sufficient stability to prevent recurrence, degradation or shrinkage [2]. To meet these criteria, in recent years more and more lightweight, large-pore meshes have been developed, while taking account of the stability needed [3]. In experimental studies, it has been demonstrated that the application of an additional coating of atomic titanium to the polypropylene filaments has resulted in further improvement of the biocompatibility and in significantly lower shrinkage rates compared with an identical polypropylene mesh without a titanium coating [4]. Despite manufacturers' claims, the differences between the various types of meshes are unproven and it is currently difficult to recommend any single mesh [1].

Therefore, the following evidence-based systematic review endeavors to evaluate all experimental and clinical studies to determine whether titanized meshes confer advantages over other synthetic meshes in hernia surgery. 


\section{Materials and methods}

A search of the medical literature from 2002 to 2012 as indexed by Medline was performed, using the PubMed search engine (http://www.pubmed.gov). To capture all potentially relevant articles with the highest degree of sensitivity, the search terms were intentionally broad. The following search terms were used: hernia mesh $(6,014$ citations), titanium coating (2,674 citations), lightweight mesh (36 citations), TiMesh (27 citations), mesh complications (6,584 citations). Moreover, a hand search of the bibliographies of relevant articles and product literature was conducted to identify additional pertinent reports. To answer the research question, it was then possible to identify 34 relevant publications.

All papers were graded according to the Oxford hierarchy of evidence as outlined below consisting of the following five levels:

1A. Systematic review of RCTs (with consistent results from individual studies).

1B. RCTs (of good quality).

2A. Systematic review of 2 B studies (with consistent results from individual studies).

2B. Prospective comparative studies (or RCT of poorer quality).

2C. Outcome studies (analysis of large registries, population-based data, etc.).

3. Retrospective, comparative studies and case-control studies.

4. Case series (i.e., studies without control group).

5. Expert opinion, animal or laboratory experiments.

For the recommendations, the following grading scale was used:

A Consistent level 1 studies $=>$ strict recommendations (standard, surgeon must do it).

B Consistent level 2 or 3 studies or extrapolations from level 1 studies = less strict wording (recommendation, surgeon should do it).

Fig. 1 Schematic representation of the PACVD technique
C Level 4 studies or extrapolation from level 2 or 3 studies $=>$ vague wording (option, surgeon can do it).

D Level 5 evidence or troublingly inconsistent or inconclusive studies at any level $=>$ (no recommendation, describe option).

Characteristics of titanized polypropylene meshes

Currently, there are two titanized polypropylene meshes in the market:

- TiMesh (pfm, Cologne, Germany)

- $\mathrm{TiO}_{2}$ Mesh (Biocer, Bayreuth, Germany)

The $\mathrm{TiO}_{2}$ mesh is a lightweight, monofilamentous polypropylene mesh with a weight of $45 \mathrm{~g} / \mathrm{m}^{2}$ and a pore size of $3.0 \mathrm{~mm}$. The polypropylene filaments are coated with titanium dioxide.

To date, no experimental or clinical studies have been carried out on this titanized polypropylene mesh. Therefore, no scientific statements can be issued regarding this mesh. Hence, the remaining literature analyses relate exclusively to the titanized polypropylene mesh TiMesh.

In the TiMesh, the polypropylene filaments are coated with a few layers of atomic titanium using what is known as the PACVD technique (plasma-activated chemical vapor deposition). The procedures performed in the coating chamber are schematically illustrated in Fig. 1.

In the coating chamber, the polypropylene filaments are coated with an approximately $30 \mu \mathrm{m}$ thick titanium layer (Fig. 2).

Depending on the tensile strength required, TiMesh ${ }^{\mathrm{TM}}$ is available in different weights based on the filament sizes used $(65,90,120 \mu \mathrm{m})$. The various characteristics of TiMesh $^{\mathrm{TM}}$ extralight, TiMesh ${ }^{\mathrm{TM}}$ light and TiMesh ${ }^{\mathrm{TM}}$ strong are shown in Table 1 (details provided by manufacturer).

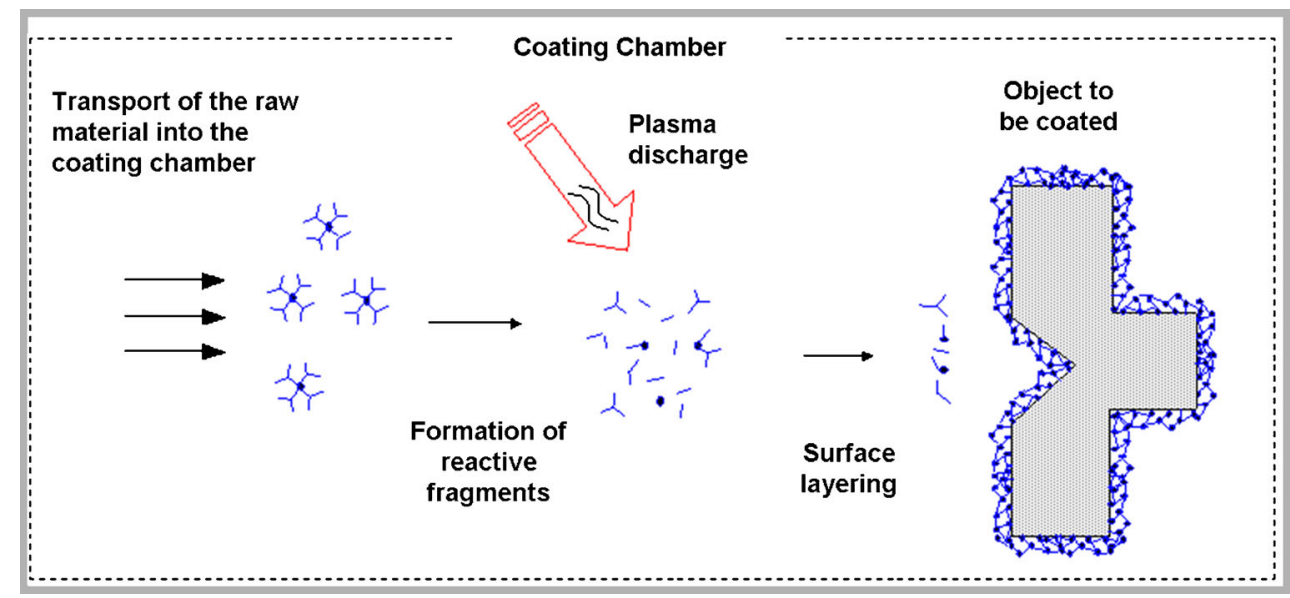




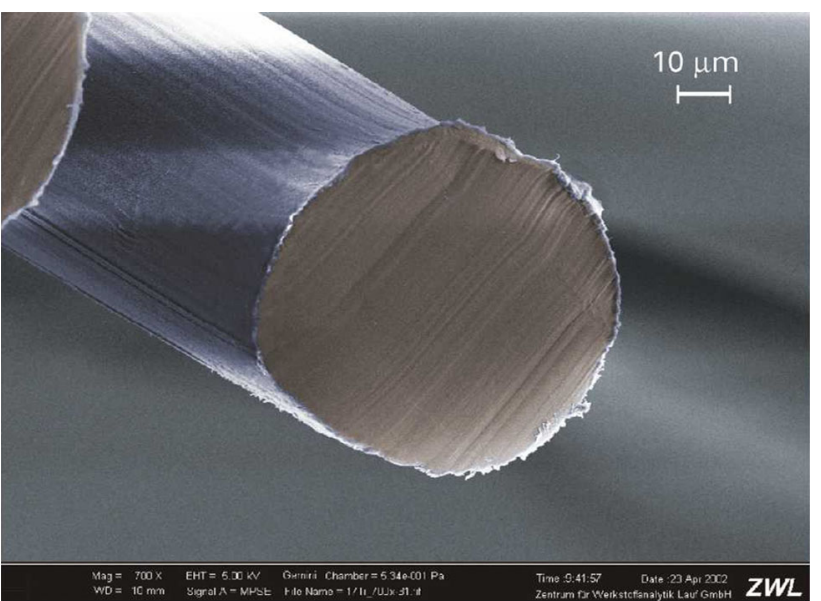

Schnitt durch titanisierten Kunststoff: monofiler Faden, REM

Fig. 2 Electronic micrograph of a cut titanium-coated polypropylene filament (magnification: $\times 700$ )

\section{Assignment to the existing mesh classifications}

Synthetic hernia meshes are classified by Earle et al. [5] on the basis of the weight and pore size (Table 2).

As such, based on the classification by Earle et al. [5], the TiMesh extralight is an ultra-lightweight, large-pore mesh; TiMesh ${ }^{\mathrm{TM}}$ light is a lightweight, large-pore mesh; and TiMesh strong is a medium-weight, large-pore mesh.

Table 1 Characteristics of TiMesh provided by manufacturer

\begin{tabular}{llll}
\hline & $\begin{array}{l}\text { TiMesh } \\
\text { extralight }\end{array}$ & $\begin{array}{l}\text { TiMesh } \\
\text { light }\end{array}$ & $\begin{array}{l}\text { TiMesh } \\
\text { strong }\end{array}$ \\
\hline Weight & $16 \mathrm{~g} / \mathrm{m}^{2}$ & $35 \mathrm{~g} / \mathrm{m}^{2}$ & $65 \mathrm{~g} / \mathrm{m}^{2}$ \\
Thickness & $0.20 \mathrm{~mm}$ & $0.30 \mathrm{~mm}$ & $0.45 \mathrm{~mm}$ \\
Pore size & $>1 \mathrm{~mm}$ & $>1 \mathrm{~mm}$ & $>1 \mathrm{~mm}$ \\
Filament diameter & $65 \mu \mathrm{m}$ & $90 \mu \mathrm{m}$ & $120 \mu \mathrm{m}$ \\
2D porosity & $73 \%$ & $61 \%$ & $53 \%$ \\
3D porosity & $91 \%$ & $87 \%$ & $82 \%$ \\
Physiological elasticity at $16 \mathrm{~N}$ & $23 \%$ & $20 \%$ & $8 \%$ \\
Breaking strength (grab test) & $37 \mathrm{~N}$ & $61 \mathrm{~N}$ & $142 \mathrm{~N}$ \\
\hline
\end{tabular}

Table 2 Categories of prosthetic pore size and density [5]

\begin{tabular}{ll}
\hline Heavy weight & $>90 \mathrm{~g} / \mathrm{m}^{2}$ \\
Medium weight & $50-90 \mathrm{~g} / \mathrm{m}^{2}$ \\
Lightweight & $35-50 \mathrm{~g} / \mathrm{m}^{2}$ \\
Ultra-lightweight & $<35 \mathrm{~g} / \mathrm{m}^{2}$ \\
Very large pore & $>2,000 \mu \mathrm{m}$ \\
Large pore & $1,000-2,000 \mu \mathrm{m}$ \\
Medium pore & $600-1,000 \mu \mathrm{m}$ \\
Small pore & $100-600 \mu \mathrm{m}$ \\
Microporous (solid) & $<100 \mu \mathrm{m}$
\end{tabular}

Synthetic meshes are classified by Coda et al. [6] on the basis of the biomaterial composition and weight. Classification based on biomaterial composition is as follows: simple (prosthetics made of one pure biomaterial; composite (prosthetics made of two or more different layers; combined (prosthetics made of two materials knitted or woven together).

In addition, the weight is used for classification (Table 3).

Based on the classification by Coda et al. [6], TiMesh is assigned to the group of combined prosthetics, TiMesh extralight to the ultra-lightweight synthetic meshes and TiMesh light and strong to the lightweight synthetic meshes.

Classification of synthetic meshes by Klinge et al. [7] is based on analysis of 1,000 explanted meshes. In addition to the weight and textile porosity, the latter introduces a further consideration, the effective porosity, into the mesh classification system. Porosity is mainly measured as the percentage of the area of the mesh which is not covered by filaments, thus reflecting the textile porosity, whereas the effective porosity represents only the area of "good" pores, where bridging of scar tissue is avoided by sufficient interfilamentary distance [7].

Klinge et al. [7] divide flat meshes with pores (meshes without pores, 3D meshes and biological meshes are assigned to a class of their own) into three classes (Table 4).

Based on these insights gleaned from 1,000 explanted meshes, TiMesh is assigned to Class I. In addition to a textile porosity of $>60 \%$, this group is endowed with an effective porosity. The clinical data show that for Class I meshes, the rate of infection and pain implicated as the reason for explantation was significantly lower compared with Class II. Quantification of the amount of inflammatory

Table 3 Categories of prosthetic density Coda et al. [6]

\begin{tabular}{ll}
\hline Ultra-light & $<35 \mathrm{~g} / \mathrm{m}^{2}$ \\
Light & $\geq 35$ to $<70 \mathrm{~g} / \mathrm{m}^{2}$ \\
Standard & $\geq 70$ to $<140 \mathrm{~g} / \mathrm{m}^{2}$ \\
Heavy & $\geq 140 \mathrm{~g} / \mathrm{m}^{2}$ \\
\hline
\end{tabular}

Table 4 Classification of flat meshes [7]

Class I Large-pore meshes characterized by a textile porosity of $>60 \%$ or an effective porosity of $>0 \%$

Class II Small pore meshes characterized by a textile porosity of $<60 \%$ and without any effective porosity

Class III Meshes with special features. This group includes porous meshes with special features to prevent adhesions as realized in meshes with barrier function for intraperitoneal use or with surface coating 
and connective tissue in the mesh area confirmed that there were significant differences between the mesh classes, with the highest values recorded for small-pore meshes or plugs and lowest values for Class I meshes [7].

The significance of the various means of mesh classification must be verified in the future on the basis of comparative clinical studies and their importance identified.

\section{Statements}

Level 5 Based on the classification by Earle et al [5], TiMesh extralight is an ultra-lightweight, large-pore mesh; TiMesh light is a lightweight, large-pore mesh; and TiMesh strong is a medium-weight, large-pore mesh

Based on the classification by Coda et al. [6], TiMesh is assigned to the group of combined prosthetics, TiMesh extralight to the ultra-lightweight synthetic meshes and TiMesh light and strong to the lightweight synthetic meshes

Based on the classification by Klinge et al. [7], TiMesh is assigned to Class I with a textile porosity of $>60 \%$ and an effective porosity

\section{Biomechanical tests}

Biomechanical tests were performed with TiMesh to investigate various issues of clinical relevance.

\section{Simulation of primary mesh augmentation for abdominal wall closure}

In the literature, the prospect of reducing the incidence of incisional hernias through primary mesh augmentation for abdominal wall closure is discussed time and again. In the experimental study conducted by Schug-Pass et al. [8] with the biomechanical model of the stamp penetration test, for a standardized incision in muscle tissue the following closure techniques were compared with each other: simple closure with single-button sutures, additional fixation with fibrin glue and augmentation of the suture with $2 \mathrm{~cm}$ overlapping TiMesh strips secured with fibrin glue.

The single suture conferred a tensile strength that was just above the prescribed maximum abdominal pressure of $32 \mathrm{~N}(37.3 \mathrm{~N})$. The additional use of fibrin glue did not have any significant impact on these results $(41.8 \mathrm{~N})$. Only through mesh augmentation with fibrin glue fixation was it possible to achieve a significantly greater tensile strength $(64.5 \mathrm{~N} ; p=0.003)$.

\section{Simulation of mesh fixation with glue in inguinal hernia surgery}

Likewise with the stamp penetration test, Schug-Pass et al. [9] investigated the fixation strength of various meshes that had been fixed to muscle tissue with the fibrin glue Tissucol/Tisseel. Six different lightweight meshes were tested: TiMesh light, TiMesh extralight, Parietene light, Ultrapro, Optilene LP and Bard Soft Mesh. Two milliliters of Tissucol was used for fixation. Five meshes from each group were tested on muscle tissue with and without fibrin glue. The defined defect was $4.5 \mathrm{~cm}$ in diameter. The biomechanical measurements were taken in a standardized way using a materials testing machine. The minimum fixation strength required was $32 \mathrm{~N}$, calculated from a corresponding model. The fixation strength measurements without fibrin glue gave a mean value for all 30 meshes of $2.98 \mathrm{~N}$ with an SD of $0.92 \mathrm{~N}$. This was far below the $32 \mathrm{~N}$ required. With fibrin glue, the mean of all the measurements ( 30 meshes) was $61.86 \pm 23.0 \mathrm{~N}$. The lowest value was recorded for Ultrapro $(34.9 \pm 12.5 \mathrm{~N})$. All the other meshes had a significantly higher fixation strength when fixed with fibrin glue than did Ultrapro $(p=0.001)$. The best results were obtained for Optilene LP, which performed significantly better than all the other meshes $(97.3 \pm 8.9 \mathrm{~N} ; p<0.001)$.

In a further biomechanical study, Schug-Pass et al. [10] investigated with which combination of mesh and fibrin glue the best fixation strength to muscle tissue could be achieved. Three different lightweight polypropylene meshes (TiMesh light, Ultrapro, Optilene LP) were tested. All meshes were fixed using $2 \mathrm{ml}$ of each of the three different fibrin glues (Tissucol, Quixil, Evicel) and tested for their biomechanical stability. The defect size in the muscle tissue used was $45 \mathrm{~mm}$ for a mesh size of $10 \times 15 \mathrm{~cm}$. Measurements were conducted using a standardized stamp penetration test, while aiming not to use a fixation strength of less than $32 \mathrm{~N}$. TiMesh light generated the best fixation strength of $64.3 \pm 8.9 \mathrm{~N}$ with Evicel, Optilene LP of $97.3 \pm 8.9 \mathrm{~N}$ with Tissucol/Tisseel and Ultrapro of $114.7 \pm 15.5 \mathrm{~N}$ with Evicel. This study shows that there were significant differences in the fixation strength achieved for different polypropylene meshes in combination with the various fibrin glues. The experiments demonstrate that for each mesh there is an optimum combination with a particular fibrin glue with respect to the fixation strength. Titanization of polypropylene apparently did not give rise to any improvement in the fixation strength on using fibrin glue, since the maximum fixation strength values measured for the other meshes with their optimum fibrin glue combination were significantly higher. Nonetheless, the fixation strength values obtained were sufficient for TiMesh too, since these were double $(64.3 \pm 8.9 \mathrm{~N})$ the maximum abdominal pressure values $(32 \mathrm{~N})$.

As an alternative to fibrin glue, there is an increasing trend toward using synthetic glues for mesh fixation in both open and endoscopic hernia surgery. Therefore, studies are 
needed to compare the fixation strength of (semi-) synthetic glues with that of fibrin glue. Schug-Pass et al. [11] used the biomechanical model to compare the adhesive strength of the two surgical glues (BioGlue and Glubran II) with the fibrin glue Evicel. They used the lightweight polypropylene mesh TiMesh light. In each case, the biomechanical stability of five meshes in each group was tested with $2 \mathrm{ml}$ fibrin glue (Evicel), $2 \mathrm{ml}$ Bioglue or $2 \mathrm{ml}$ GlubranII (cyanoacrylate). The defect in the muscle tissue used was $4.5 \mathrm{~cm}$ in diameter for a mesh size of $10 \times 15 \mathrm{~cm}$. Measurements were taken using a standardized stamp penetration test, while aiming not to remain under a minimum fixation strength of $32 \mathrm{~N}$.

Using Evicel for mesh fixation, an adhesive strength of 64.3 N was achieved. Using Glubran II, it was possible once again to significantly improve the adhesive strength $(105.4 \mathrm{~N}, p=0.008)$. The use of BioGlue improved the adhesive strength to $131.7 \mathrm{~N}$, but not significantly so, compared with Glubran II $(p=0.110)$.

In conclusion, in terms of adhesive strength, (semi-) synthetic glues can be used for mesh fixation instead of fibrin glue and even achieve significantly better adhesive strength than fibrin glue. pressure of $32 \mathrm{~N}$, fixation of TiMesh with fibrin glue to the peritoneum was not adequate.

In another biomechanical test, Rieder et al. [13] investigated the tangential detachment forces (TF) of various meshes that had been fixed with fibrin glue to the abdominal wall, with peritoneum, of pigs. That was compared with mesh fixation with tacks.

Tangential detachment forces tests revealed that fibrin glue attachment was not substantially different from that achieved with absorbable tacks (median TF $7.8 \mathrm{~N}$, range 1.3-15.8 N), but only when certain open porous meshes (polyvinylidene fluoride/polypropylene mesh: median $6.2 \mathrm{~N}$, range 3.4-10.3 N; titanium-coated polypropylene mesh: median 5.2 N, range 2.1-11.7 N) were used. Meshes coated with an anti-adhesive barrier (polypropylene/polydioxanone mesh: median $3.1 \mathrm{~N}$, range 1.7-5.8; polyester mesh bonded with an absorbable collagen: median $1.3 \mathrm{~N}$, range $0.5-1.9 \mathrm{~N}$ ) or the condensed PTFE mesh (median $3.1 \mathrm{~N}$, range 2.1-7.0 N) provided a significantly lower TF $(p<0.01)$.

In conclusion, in a biomechanical model with tangential detachment forces, fibrin glue appears to be an appealing noninvasive option for mesh fixation in laparoscopic ventral hernia repair, but only if appropriate meshes are used.

Statements

Level 5 In a biomechanical model, it was possible to achieve a greater tensile strength for a single sutured muscle tissue incision through mesh augmentation with TiMesh fixed with fibrin glue

In a biomechanical model, it can be demonstrated that, on using fibrin glue, it is possible to fix pure polypropylene meshes, polypropylene meshes with an absorbable portion of polyglecaprone and titanized polypropylene meshes with adequate tensile strength to muscle tissue. However, there are significant differences between the meshes. There is always an optimum combination of a particular mesh and corresponding fibrin glue. For TiMesh the best fixation strength was conferred by Evicel

In a biomechanical model, it can be demonstrated that the fixation strength achieved for TiMesh with fibrin glue to peritoneum is not adequate

In a biomechanical model, it can be demonstrated that fixation of TiMesh with fibrin glue to peritoneum generates tangential detachment forces comparable to those obtained for fixation with absorbable tacks

In a biomechanical model, it can be demonstrated that the fixation strength of TiMesh to muscle tissue with the (semi-) synthetic glues Glubran II and BioGlue is greater than that achieved with the most effective fibrin glue Evicel

\section{Simulation of mesh fixation to the peritoneum with fibrin} glue

To elucidate the fixation strength von TiMesh light with fibrin glue to the peritoneum, Schug-Pass et al. [12] carried out a further stamp penetration test with the biomechanical model, using muscle tissue with peritoneum compared to muscle tissue without peritoneum. Here, too, the defect diameter was $45 \mathrm{~mm}$ and mesh size $10 \times 15 \mathrm{~cm}$. The fibrin glue used was $2 \mathrm{ml}$ Tissucol/Tisseel. The fixation strength of the mesh to the muscle tissue with peritoneum, at $11.86 \pm 3.89 \mathrm{~N}$, was significantly lower than that achieved to muscle tissue, at $47.88 \pm 10.89 \mathrm{~N}(\mathrm{p}=0.001)$. Since, as such, the fixation strength to peritoneum was below the maximum abdominal
Animal experimental tests

The closest models to surgical practice are those using large animals, swine or sheep, which allow construction of hernias that resemble human anatomy. Rats or rabbits have thus been used particularly to evaluate the tissue reaction of different materials and for biomechanical testing of the healed tissue [14]. Because of these differences, it is beneficial to present the results of animal experiments in relation to the model used.

\section{Small animal models}

Mesh placement in the abdominal wall To analyze the pure effect of titanium coating, two different mesh 
structures were studied by Junge et al. [15] using a standardized rat animal model. The titanium-coating, monofilamentous, large porous and lightweight mesh made of polypropylene and coated with titanium (TiMesh light) was compared to a pure polypropylene mesh manufactured with a similar structure and amount of material serving as a control. The mesh samples were placed in a subcutaneous position. Both mesh modifications investigated showed overall good biocompatibility after 182 days. Macroscopic clinical observation was uneventful. The tissue response to the polypropylene as well as to the titanized polypropylene was characterized by a moderate inflammatory tissue reaction limited to the perifilamentary region, as is known for low-weight, large porous and monofilamentous mesh structures. No significant improvement of biocompatibility was found when analyzing the effect of titanium coating compared to the pure polypropylene mesh structure.

Another study by Pereira-Lucena et al. [16] aimed to compare the serum and tissue inflammatory responses and collagen deposition caused by meshes made of polypropylene, polypropylene and polyglactin and titanized polypropylene. The meshes were positioned on the abdominal wall of rats. The pro-inflammatory cytokines were assayed postoperatively. On the 40th postoperative day in the group with the high-density polypropylene mesh, there were fewer inflammatory tissue responses and greater collagen deposition $(p<0.01)$. In group II with polypropylene mesh plus polyglactin, there were greater inflammatory tissue responses and less collagen deposition $(p>0.01)$. The polypropylene plus titanium coating group produced intermediate values between the others.

Intraperitoneal mesh placement In a rat study by Burger et al. [17], eight different meshes were placed intraperitoneally and in direct contact with abdominal viscera. The following meshes were tested: polypropylene (Prolene), e-PTFE (Dualmesh), poylproylene-polyglecaprone composite (Ultrapro), titanium-polypropylene composite (TiMesh), polypropylene with carboxymethylcellulosesodium hyaluronate coating (Sepramesh), polyester with collagen-polyethylene glycol-glycerol coating (Parietex composite), polypropylene-polydiaxanone composite with oxidized cellulose coating (Proceed) and bovine pericardium (Tutomesh). At 7 and then 30 days postoperatively, adhesion formation, mesh incorporation, tensile strength, shrinkage and infection were scored by two independent observers.

Parietex composite, Sepramesh and Tutomesh resulted in decreased surface coverage with adhesions, whereas Prolene, Dualmesh, Ultrapro, TiMesh and Proceed resulted in increased adhesion coverage. Parietex composite, Prolene, Ultrapro and Sepramesh resulted in the greatest mesh incorporation. Dualmesh and Tutomesh resulted in significantly increased shrinkage. There were no differences in mesh infection. Parietex composite and Dualmesh resulted in a moderate inflammatory reaction, as compared to the mild reaction the other meshes exhibited. In conclusion, Parietex composite and Sepramesh combine minimal adhesion formation with maximum mesh incorporation and tensile strength.

The purpose of another study by Chatzimavroudis et al. [18] was to identify any differences in the systemic inflammatory response after the intraperitoneal implantation of three different types of polypropylene mesh (pure polypropylene mesh, BardMesh; titanium-coated polypropylene mesh, TiMesh; and composite polypropylene/e-PTFE mesh, Bard composix mesh) in rabbits. Statistically significant elevations of white blood cell count, tumor necrosis factor-alpha and malondialdehyde were observed in all groups postoperatively $(p<0.05)$. There were no statistically significant differences between the mesh groups. In conclusion, intraperitoneal mesh implantation induces a mild systemic inflammatory response regardless of the type of implanted mesh.

In a rat study by Schreinemacher et al. [19], six commercially available meshes were placed intraperioneally against a closed peritoneum: Prolene (polypropylene), TiMesh (polypropylene composite with titanium), Ultrapro (polypropylene composite with polyglecaprone), Proceed (polypropylene mesh coated with a layer of cellulose), Parietex composite (polyester with a layer of collagen) and C-Qur (polypropylene mesh coated with a layer of omega3 fatty acids). Parietex composite and C-Qur significantly reduced adhesion formation at 7 days follow-up compared with all other meshes. By 30 days, this effect had diminished with a significant increase in adhesions together with phagocytosis of the coating seen for all meshes with layered coatings (Proceed, Parietex composite and C-Qur). Incorporation was insufficient for all meshes. In conclusion, the absorbable layers of Parietex composite and C-Qur reduce adhesion formation to the intraperitoneal mesh in the short term, but the effect diminishes and phagocytosis of absorbable coatings may contribute to adhesion formation.

In a rat study by Ott et al. [20], conventional (Premilene) and titanium-coated polypropylene meshes (TiMesh) were implanted under the abdominal wall employing the inlay technique. Implantation of the meshes was performed under semi-sterile conditions and bacterial contamination of the meshes. The meshes were explanted after 28 days. All the materials implanted under semi-sterile conditions were incorporated into the abdominal wall with only a few intraabdominal adhesions. In the bacterial contamination group, all meshes were associated with a suppurating infection and strong adhesions between the bowel and mesh. In conclusion, irrespective of the material employed, implantation of alloplastic meshes in an abdominal wall 
contaminated with bacteria is associated with suppurating infections.

Fortelny et al. [21] investigated the integration of von TiMesh into the abdominal wall of rats after fixing the mesh with the synthetic glue Glubran II. They found that tissue integration of TiMesh was impaired by impenetrable glue plaques. At application sites, the elasticity of the abdominal wall was significantly reduced because of nonabsorbed, rigid glue residues.

They concluded that mesh fixation with Glubran II impairs tissue integration, elicits inflammation and unfavorably alters the biomechanics of a macroporous mesh and the abdominal wall.

Statements

Level 5 In a small animal model, intraperitoneal mesh implantation induces mild systemic inflammatory response regardless of the type of implanted mesh

In a small animal model with intraperitoneal mesh placement, TiMesh showed increased adhesion coverage and mild inflammatory reaction in comparison to Parietex composite and Sepramesh

In a small animal model, irrespective of the material employed, implantation of alloplastic meshes in an abdominal wall contaminated with bacteria is associated with suppurating infections

In a small animal model, tissue integration of TiMesh fixed with cyanoacrylate was impaired by impenetrable glue plaques

In a small animal model, no significant improvement of biocompatibility was found when analyzing the effect of titanium coating compared to the pure polypropylene mesh structure

\section{Large animal models}

Inguinal hernia repair In a comparative study by Scheidbach et al. [4], totally endoscopic extraperitoneal patch plasty (TEP) was performed on 11 pigs in each of the two groups. In one group, an Atrium mesh was implanted during TEP and a titanium-coated Atrium mesh in the other group. A significant difference in the shrinkage behavior was noted between conventional Atrium and titaniumcoated Atrium meshes (14.9 vs. $8.8 \%, p<0.05)$. Furthermore, the partial volume of the inflammatory infiltrate also proved to be smaller with the titanium-coated mesh (14.9 vs. $12.4 \%$ ). Besides, Ki-67 expression was lower in the group implanted with titanium-coated mesh (21.0 vs. $15 \%)$. No difference was observed with regard to the apoptosis index (7.6 vs. 6.5 ). The authors concluded that titanium-coated polypropylene mesh induced a less pronounced foreign body reaction in comparison with identical meshes with no titanium coating.
In another comparative study by Scheidbach et al. [22], following TEP operations on a porcine model a shrinkage rate of $12 \%$ was observed for Atrium, $28 \%$ for Vypro II, $7 \%$ for Parietene and $5 \%$ for TiMesh extralight. The authors concluded that titanium coating of the TiMesh conferred an additional advantage in terms of biocompatibility.

Ventral hernia repair In an animal experimental study by Schug-Pass et al. [23] involving laparoscopic IPOM operation, TiMesh was compared with Dualmesh made of expanded polytetrafluoroethylene (e-PTFE). Six pigs each underwent laparoscopic intraabdominal placement of either a TiMesh light or a Dualmesh. With the titanium-coated polypropylene meshes, the average total adhesion area was only 0.085 , as compared to 0.25 for the e-PTFE mesh $(p=0.055)$. The Dualmesh showed an average shrinkage to almost half of the original surface area (median 0.435). The average shrinkage of the TiMesh was to 0.18 of the original area $(p=0.006)$, which was significantly smaller. Determination of the partial volume of the inflammatory cells showed significantly lower median values for TiMesh $(p=0.009)$. Measurements of the proliferation marker Ki67 showed significantly higher values for e-PTFE than for TiMesh $(p=0.011)$. The apoptosis index was significantly higher for the e-PTFE membranes $(p=0.002)$. The authors concluded that titanium-coated polypropylene mesh was clearly superior to the e-PTFE mesh in terms of biocompatibility and thus suitable for the laparoscopic intraperitoneal repair of abdominal wall hernias.

A further study by Schug-Pass et al. [24] aimed to determine whether the additional application of an adhesion-barrier substance resulted in a further reduction in adhesions and shrinkage of intraperitoneally implanted titanized meshes. Using the laparoscopic intraperitoneal onlay mesh technique, six pigs were implanted with either a lightweight polypropylene mesh (TiMesh light) or TiMesh plus an adhesion-barrier film made of polylactide (SurgiWrap). No adhesions to intestinal structures were found in any of the animals. Adhesions between the greater omentum and the mesh did not differ significantly between the TiMesh (32\%) and TiMesh with SurgiWrap (33.5\%) groups. Shrinkage of the mesh's surface area was comparable between the two groups (18 vs. $21 \%$ ). Histology showed pronounced inflammatory reaction and bridging of scar tissue between the filaments with the use of SurgiWrap. In conclusion, the additional application of a slowly absorbable adhesion-barrier film made of polylactide (SurgiWrap) does not appear to confer any further benefit.

Another study by Schug-Pass et al. [12], involving laparoscopic IPOM operation on a porcine model, aimed to identify whether adequate fixation of TiMesh light to the peritoneum could be achieved with fibrin glue alone. The mesh was positioned in the middle/upper abdomen and 
fixed to the peritoneum by spray application of $2 \mathrm{ml}$ of fibrin glue (Tissucol/Tisseel). After 4 months, three of the implanted meshes were not completely integrated, and two of three were dislocated. In conclusion, mesh fixation with fibrin glue alone to the undamaged peritoneum in the abdomen cannot be recommended because of the risk of dislocation and incomplete integration.

\section{Statements}

Level 5 In a large animal model that had undergone TEP operation, a titanized Atrium mesh exhibited a significantly lower shrinkage rate, as well as a less pronounced foreign body reaction, compared with that of an Atrium mesh alone

In a large animal model that had undergone TEP operation, a significantly lower shrinkage rate was observed for TiMesh extralight compared with Vypro II and Parietex

In a large animal model that had undergone laparoscopic IPOM operation, a significantly lower shrinkage rate was observed for TiMesh light, as well as significantly better biocompatibility, compared with ePTFE-mesh Dualmesh

In a large animal model that had undergone laparoscopic IPOM operation, it can be demonstrated that the additional application of a slowly absorbable adhesionbarrier film made of polylactide does not confer any benefits

In a large animal model that had undergone laparoscopic IPOM operation, it can be demonstrated that fixation of TiMesh with fibrin glue alone to the undamaged peritoneum cannot be recommended because of the risk of dislocation and incomplete integration

\section{Clinical studies}

Randomized, controlled trials, when appropriately designed, conducted and reported, represent the gold standard in evaluating health-care interventions. However, randomized trials can yield biased results if they lack methodological rigor [25].

Based on the CONSORT (Consolidated Standards of Reporting Trials) statements, the quality of prospective, randomized, controlled comparative trials can be evaluated. Therefore, the quality of the prospective, randomized, controlled trials discussed below was first verified and evaluated on the basis of the CONSORT statements. All studies proved to be of very good quality, but with weaknesses in certain criteria (Table 5). One drawback is the lack of blinding of the investigator entrusted with re-evaluation of highly investigator-dependent outcome criteria.

\section{Inguinal hernia repair}

In a prospective, randomized controlled monocenter trial by Koch et al. [26], 317 patients with an inguinal hernia were operated on with the Lichtenstein technique under general anesthesia. Surgery was conducted for 161 patients with the $80 \mathrm{~g} / \mathrm{m}^{2}$ polypropylene mesh Prolene and for 156 patients with the lightweight titanized polypropylene mesh TiMesh light with a weight of $35 \mathrm{~g} / \mathrm{m}^{2}$. Both meshes measured $10 \times 15 \mathrm{~cm}$.

Pain before and after surgery, and during convalescence (primary outcome) was estimated. At 1-year clinical follow-up, recurrence, pain, discomfort and quality of life (secondary outcome) were evaluated. Patients with the lightweight TiMesh returned to work after 4 days, compared with 6.5 days for the standard mesh Prolene $(p=0.04)$. The lightweight TiMesh group returned to normal activity after 7 days vs. 10 days for the Prolene group $(p=0.005)$. There was no difference in postoperative pain and recurrence at the 1-year follow-up. The authors concluded that patients with inguinal hernias operated on with the Lichtenstein technique performed with the lightweight TiMesh had a shorter convalescence than those with the $80 \mathrm{~g} / \mathrm{m}^{2}$ polypropylene mesh Prolene.

Another prospective, randomized, single-blinded, monocenter comparative trial by Schopf et al. [27] studied the incidence of chronic pain after laparoscopic transabdominal preperitoneal hernia repair (TAPP) using a $35 \mathrm{~g} /$ $\mathrm{m}^{2}$ titanized polypropylene mesh (TiMesh light) and a $16 \mathrm{~g} / \mathrm{m}^{2}$ titanized polypropylene mesh (TiMesh extralight). Three hundred and eighty patients with 466 inguinal hernias underwent surgery. Mesh fixation with two to six titanium staples was carried out routinely. After dissection was completed just prior to the implantation of the mesh, patients were randomized into two groups. In group A, 250 $(53.6 \%)$ inguinal hernias were repaired with a $35 \mathrm{~g} / \mathrm{m}^{2}$ titanized polypropylene mesh, and in group B, 216 $(46.4 \%)$ inguinal hernias were repaired with a $16 \mathrm{~g} / \mathrm{m}^{2}$ titanized polypropylene mesh. The primary outcome was chronic pain 3 years after surgery. The degree of pain was determined using a visual analog scale (VAS) with a range from 0 to 10 . The secondary outcome was the rate of recurrence.

The postoperative period of observation was at least 3 years for every patient. In both groups, $90 \%$ of the patients could be interviewed and examined clinically: in group A with TiMesh light $\left(35 \mathrm{~g} / \mathrm{m}^{2}\right) 5.3 \%$ of the patients and in group B with TiMesh extralight $\left(16 \mathrm{~g} / \mathrm{m}^{2}\right) 1.5 \%$ of the patients suffered from chronic pain $(p=0.037)$. There was no difference with respect to the rate of recurrence: for group A it was $3.1 \%$ and for group B $2.6 \%(p=0.724)$.

In conclusion, reducing the material load of TiMesh from 35 to $16 \mathrm{~g} / \mathrm{m}^{2}$ seems to further improve the biocompatibility, thus improving the clinical outcome by reducing chronic pain to a rare event. There was no evidence supporting the notion that the use of the $16 \mathrm{~g} / \mathrm{m}^{2}$ 
Table 5 Consort checklist of information to include when reporting a randomized trial [25]

\begin{tabular}{|c|c|c|c|c|c|c|c|c|}
\hline Section/topic & $\begin{array}{l}\text { Item } \\
\text { number }\end{array}$ & $\begin{array}{l}\text { Koch et al. } \\
{[26]}\end{array}$ & $\begin{array}{l}\text { Schopf } \\
\text { et al. [27] }\end{array}$ & $\begin{array}{l}\text { Bittner et al. } \\
\text { [28] }\end{array}$ & $\begin{array}{l}\text { Fortelny } \\
\text { et al. [29] }\end{array}$ & $\begin{array}{l}\text { Peeters et al. } \\
{[30]}\end{array}$ & $\begin{array}{l}\text { Peeters et al. } \\
\text { [31] }\end{array}$ & $\begin{array}{l}\text { Moreno-Egea } \\
\text { et al. [34] }\end{array}$ \\
\hline Titel and abstract & 1 & + & + & + & + & + & + & + \\
\hline Introduction & 2 & + & + & + & + & + & + & + \\
\hline Trial design & 3 & + & + & + & + & + & + & + \\
\hline Participants & 4 & + & + & + & + & + & + & + \\
\hline Interventions & 5 & + & + & + & + & + & + & + \\
\hline Outcomes & 6 & + & + & + & + & + & + & + \\
\hline Sample size & 7 & + & + & + & + & - & - & + \\
\hline $\begin{array}{l}\text { Randomization } \\
\text { Sequenz generation }\end{array}$ & 8 & + & + & + & + & + & + & + \\
\hline $\begin{array}{l}\text { Allocation } \\
\text { concealment } \\
\text { mechanism }\end{array}$ & 9 & + & + & + & + & + & + & + \\
\hline Implementation & 10 & + & + & + & + & + & + & + \\
\hline Blinding & 11 & $\begin{array}{r}\text { Patient and } \\
\text { examiner }\end{array}$ & $\begin{array}{l}\text { Only } \\
\text { patient }\end{array}$ & $\begin{array}{l}\text { Patient and } \\
\text { examiner }\end{array}$ & No blinding & $\begin{array}{r}\text { Patient and } \\
\text { examiner }\end{array}$ & $\begin{array}{r}\text { Patient and } \\
\text { examiner }\end{array}$ & Only patient \\
\hline Statistical methods & 12 & + & + & + & + & + & + & + \\
\hline Participant flow & 13 & + & + & + & + & + & + & + \\
\hline Recruitment & 14 & - & + & - & - & + & + & + \\
\hline Baseline data & 15 & + & + & + & + & + & + & + \\
\hline Numbers analyzed & 16 & + & + & + & + & + & + & + \\
\hline $\begin{array}{l}\text { Outcomes and } \\
\text { estimation }\end{array}$ & 17 & + & + & + & + & + & + & + \\
\hline Ancillary analyses & 18 & - & - & - & - & - & - & - \\
\hline Harms & 19 & + & - & + & + & + & + & + \\
\hline Limitations & 20 & + & - & + & - & + & + & + \\
\hline Generalizability & 21 & + & + & + & + & + & + & + \\
\hline Interpretation & 22 & + & + & + & + & + & + & + \\
\hline Registration & 23 & - & - & - & + & + & + & - \\
\hline Protocol & 24 & - & - & - & - & - & - & - \\
\hline Funding & 25 & + & + & - & - & + & + & + \\
\hline
\end{tabular}

titanized meshes is associated with an increased recurrence rate.

Another prospective randomized controlled trial by Bittner et al. [28] compared the results obtained, after laparoscopic inguinal hernia operation using the TAPP technique, for ultra-lightweight titanized polypropylene meshes (TiMesh extralight) and for a standard polypropylene mesh (Prolene). The titanized mesh was not fixed, and the heavy-weight polypropylene mesh Prolene was fixed with two absorbable sutures. Three hundred patients with an inguinal hernia and a defect diameter $\leq 3 \mathrm{~cm}$ were included in the trial. Patients were assessed for pain, foreign body sensation and physical activities preoperatively, early postoperatively, at 4 weeks, 6 months and 1 year by questionnaire and examined clinically. Postoperatively, seroma formation was measured by ultrasound.

One year after TAPP, the frequency of chronic pain was not greater than $3 \%$, with no difference between the two mesh groups. In the early postoperative period, $40 \%$ of the patients in the titanized mesh group needed pain medication compared with $52.7 \%$ in the group of the standard polypropylene mesh $(p=0.0378)$. Foreign body sensation did not differ between the groups, but there was significantly less impairment of physical activities $(p=0.0425)$ and seroma production ( $p=0.0415$ ) in the titanized polypropylene mesh group compared with the standard polypropylene mesh group in the early postoperative period.

In conclusion, the use of a titanized polypropylene mesh for laparoscopic hernia repair did not affect the rate of chronic pain, but it seemed to improve early postoperative convalescence. Its use without any fixation can be recommended in TAPP for inguinal hernia patients with a defect size $\leq 3 \mathrm{~cm}$.

In another prospective randomized controlled trial by Fortelny et al. [29], 87 patients with 110 inguinal hernias were operated on with the TAPP technique. TiMesh 
extralight $\left(16 \mathrm{~g} / \mathrm{m}^{2}\right)$ was used for lateral hernias and TiMesh light $\left(35 \mathrm{~g} / \mathrm{m}^{2}\right)$ for medial hernias. In group A for 44 patients with 54 inguinal hernias, the titanized polypropylene mesh was fixed with fibrin glue (Tissucol/Tisseel), and in group B for 45 patients with 56 inguinal hernias, fixation was done with a titanium stapler (EMS ${ }^{\mathrm{TM}}$ Stapler). The observation period was 1 year with regular clinical checkups and assessment of VAS and SF-36. In each group there was one postoperative recurrence. After 1 year, there was no significant difference between the two groups with respect to the parameter pain in the SF-36 and VAS.

In conclusion, fibrin sealant fixation of titanized polypropylene meshes in the TAPP technique leads to a low rate of hernia recurrence and prevents tissue trauma. Tacker fixation produces similar results in the hands of experts, but poses inherent risks of complications due to tissue perforation.

In another prospective randomized trial by Peeters et al. [30], the quality of life and fertility aspects after repair of uni- and bilateral inguinal hernias with the TEP technique using lightweight meshes and heavy-weight meshes were compared. Twenty patients were implanted with Marlex meshes (95 g/m 2 , $1 \mathrm{~mm}$ pore size), 20 patients with Vypro II meshes (30 g/qm ${ }^{2}, 3-4 \mathrm{~mm}$ pore size) and 19 patients with TiMesh light meshes ( $35 \mathrm{~g} / \mathrm{m}^{2}, \geq 1 \mathrm{~mm}$ pore size). All meshes measured $15 \times 13 \mathrm{~cm}$. The mesh was fixed only in selected cases, e.g., large direct hernia, with a spiral tacker (Protack). In the case of bilateral hernias, identical meshes were used on both sides. Primary outcomes were male fertility aspects, measured by semen analysis and scrotal ultrasound 1 year after surgery. Patients were clinically reevaluated 1, 3, 6 and 12 months after surgery, and the secondary outcomes were documented. These outcomes included hernia-related complications, the duration to resumption of normal activities (daily, professional, and sports activities), patients' quality of life (SF-36) and pain status and hernia recurrence, clinically evaluated by an experienced surgeon. The evaluator and patients were blinded as to which mesh was implanted.

Patients implanted with a Vypro II or TiMesh exhibited decreased sperm motility (vs. preoperatively) compared with Marlex patients, $-9.5 \%$ (Vypro II) and $-5.5 \%$ (TiMesh) vs. $+2 \%$ (Marlex $)(p=0.013)$. When the results after uni- and bilateral hernia repair were analyzed separately, this difference only remained significant in the bilateral hernia subgroup: $-10 \%$ for Vypro II and $-17 \%$ for TiMesh vs. $+1 \%$ for Marlex $(p=0.037)$. Other fertility parameters (sperm concentration, morphology, and $\alpha$-glucosidase level) were unchanged. There were no differences at any study point between the three groups regarding quality of life. Only for resumption of sport activities was a small advantage noted for Vypro II vs. Marlex patients $(p=0.045)$. After 1 year, no recurrence was observed; three patients $(6 \%)$ complained of chronic disabling pain.

The authors concluded that the data suggest that the use of lightweight meshes for laparoscopic inguinal hernia repair in male patients negatively influences sperm motility, without any benefit for quality of life. These findings might be important in a subgroup of young male patients operated on laparoscopically for a bilateral hernia.

The patients in this study underwent follow-up examination by Peeters et al. [31] after a median of 39.1 months. The decrease in sperm motility in patients operated on using a lightweight mesh compared to patients operated on using a heavy-weight mesh 1 year after laparoscopic inguinal hernia repair could not be confirmed at 3 years' follow-up. Furthermore, heavy-weight and lightweight groups were comparable regarding quality of life, chronic pain and recurrence rate.

In a prospective comparative study, Horstmann et al. [32] compared in 672 consecutive patients the results of TAPP technique for primary repair of inguinal hernias using different meshes. For 232 patients a heavy-weight Prolene mesh was used, for 217 patients the lightweight, partially absorbable Vypro II mesh and for 223 patients the ultra-lightweight TiMesh extralight mesh. The groups were compared in terms of postoperative complications (seroma, wound healing disorders), quality of life score (pain development, physical condition, urologic disorders) and hernia recurrence.

During a 12-month follow-up period, there were no significant differences in the recurrence rate $(1.3-1.7 \%)$. Patients with a heavy-weight pure polypropylene mesh showed significantly more postoperative seromas (12.1 vs. $4.1 \% / 1.8 \%$ ), foreign body sensations (9.1 vs. $5.5 \% /$ $3.5 \%$ ) and sensitivity to weather changes (5.6 vs. $3.2 \%$ / $2.2 \%$ ) compared to groups with Vypro II and TiMesh extralight. In all groups, the quality of life score was improved postoperatively. However, among those patients with few preoperative complaints, the postoperative quality of life deteriorated when heavy-weight polypropylene meshes were used, but significantly improved when lightweight titanized polypropylene meshes were used.

In a case series of 11 patients, Fortelny et al. [33] investigated the quality of life before and after inguinal hernia surgery using the TAPP technique and mesh fixation with fibrin glue. TiMesh extralight $\left(16 \mathrm{~g} / \mathrm{m}^{2}\right)$ was used for lateral hernias and TiMesh light $\left(35 \mathrm{~g} / \mathrm{m}^{2}\right)$ for medial hernias. Twelve months after surgery, a strikingly significant improvement was detected in physical health and pain reduction.

\section{Ventral hernia repair}

Moreno-Egea et al. [34] performed a randomized controlled monocenter clinical trial using the basic principle of 
one unit, one surgeon and one technique (midline incisional hernia with a laparoscopic approach). He compared the use of a lightweight titanium-coated polypropylene mesh (TiMesh light) and a medium-weight collagen-polyester composite mesh (Parietex composite) in 102 patients (51 patients in each arm). The primary end points were pain and recurrence. The secondary end points were morbidity and patient outcomes (analgesic consumption, return to everyday activities). The postoperative complication rates were similar for the two meshes. Pain was significantly less activities after 6.9 vs. 9.7 days for the Parietex composite group $(p>0.001)$. The rate of recurrence did not differ between the two groups at the 2-year follow-up evaluation.

In conclusion, the lightweight titanium-covered polypropylene mesh was associated with less postoperative pain in the short term, lower analgesic consumption and a quicker return to everyday activities than the Parietex composite medium-weight mesh. The recurrence rates at 2 years showed no difference between the two groups.

Statements on inguinal hernia repair

1B In a prospective, randomized controlled trial it was demonstrated that patients with inguinal hernias operated on with the Lichtenstein technique performed with the lightweight TiMesh have a shorter convalescence than those with the heavy-weight mesh Prolene

In a prospective, randomized controlled trial with patients who had had an inguinal hernia repaired with the TAPP technique, it was demonstrated that by reducing the material load of TiMesh from 35 to $16 \mathrm{~g} / \mathrm{m}^{2}$ the biocompatibility seemed to be further improved, in turn improving the clinical outcome by reducing chronic pain to a rare event without increased recurrence rate

In a prospective, randomized controlled trial, it was demonstrated that in patients with inguinal hernia operated on with the TAPP technique with the lightweight TiMesh in comparison to the heavy-weight Prolene mesh, the early postoperative convalescence seems to improve. Its use without any fixation can be recommended in TAPP for inguinal hernia patients with a defect size $\leq 3 \mathrm{~cm}$

In a prospective, randomized controlled trial it was demonstrated that in patients with inguinal hernia, fibrin sealant fixation of titanized polypropylene meshes in TAPP technique leads to a low rate of hernia recurrence and prevents trauma

In a prospective, randomized controlled trial it was demonstrated that in male patients 1 year after TEP operation, because of bilateral inguinal hernia, the use of TiMesh light compared with the heavy-weight Marlex negatively influences sperm motility, without any benefit on quality of life. That negative effect could no longer be detected on follow-up examination after 3 years

2B In a prospective comparative study of inguinal hernia patients operated on with the TAPP technique, a lower rate of postoperative seromas, foreign body sensations and sensitivity to weather changes without increase in recurrence rate was noted on using TiMesh extralight compared to a heavy-weight polypropylene mesh

4 In a case series of inguinal hernia operations performed with the TAPP technique and TiMesh, a strikingly significant improvement was detected in physical health and pain reduction after 12 months compared to preoperatively

Statement on ventral hernia repair

1B In a prospective, randomized controlled trial of midline incisional hernias with a laparoscopic IPOM technique, the lightweight titaniumcoated polypropylene mesh was associated with less postoperative pain in the short term, lower analgesic consumption and a quicker return to everyday activities than the Parietex composite medium-weight mesh

Recommendations

Level B Titanized polypropylene meshes can be used in inguinal hernia repair in Lichtenstein technique instead of heavy-weight pure polypropylene meshes

Level B In the TAPP technique for inguinal hernia repair, the ultra-light titanized polypropylene mesh can be preferred to heavy-weight meshes in defect sizes $\leq 3 \mathrm{~cm}$

Level B Titanized polypropylene meshes can be used for laparoscopic (TAPP) and endoscopic (TEP) repair for unilateral and bilateral inguinal hernias

Level B Titanized polypropylene meshes can be used for laparoscopic ventral hernia repair in IPOM technique

common in the TiMesh group at 1 month $(p=0.029)$, but was similar for the two groups at 6 months and 1 year. There was a significant difference between the two groups in the average use of analgesics: 1.6 days in the TiMesh group vs. 6.1 days in the Parietex composite group $(p<0.001)$. The TiMesh group returned to everyday

\section{Conflict of interest None.}

Open Access This article is distributed under the terms of the Creative Commons Attribution Noncommercial License which permits any noncommercial use, distribution, and reproduction in any medium, provided the original author(s) and the source are credited. 


\section{References}

1. Brown CN, Finch JG (2010) Which mesh for hernia repair? Ann R Coll Surg Engl 92:272-278 Level 5

2. Earle D, Romanelli J (2007) Prosthetic materials for hernia: what's new. Contemp. Surg. 63(2):63-69 Level 5

3. Scheidbach H, Wolff S, Lippert H (2011) Prothetische Materialien in der Bauchwandchirurgie-ein Überblick. Zentralbl Chir 136:568-574 Level 5

4. Scheidbach H, Tannapfel A, Schmidt U, Lippert H, Köckerling F (2004) Influence of titanium coating on the biocompatibility of a heavyweight polypropylene mesh. Eur Surg Res 36:313-317 Level 5

5. Earle DB, Mark LA (2008) Prothetic material in inguinal hernia repair: how do I choose? Surg Clin N Am 88:179-201 Level 5

6. Coda A, Lamberti R, Marorana S (2011) Classification of prosthetics used in hernia repair based on weight and biomaterial. Hernia doi:10.1007/s10029-011-0868-z (Level 5)

7. Klinge U, Klosterhalfen B (2012) Modified classification of surgical meshes for hernia repair based on the analyses of 1,000 explanted meshes. Hernia doi:10.1007/s10029-012-0913-6 (Level 5)

8. Schug-Pass C, Lippert H, Köckerling F (2010) Primary mesh augmentation with fibrin glue for abdominal wall closure: investigations on a biomechanical model. Langenbecks Arch Surg 395:151-156 Level 5

9. Schug-Pass C, Lippert H, Köckerling F (2010) Mesh fixation with fibrin glue (Tissucol/Tisseel ${ }^{\circledR}$ ) in hernia repair dependent on the mesh structure: is there an optimum fibrin-mesh combination? Investigations on a biomechanical model Langebecks. Arch Surg 395:569-574 Level 5

10. Schug-Pass C, Jacob DA, Lippert H, Köckerling F (2012) Differences in biomechanical stability using various fibrin glue compositions for mesh fixation in endoscopic inguinal hernia repair. Surg Endosc 26:3282-3286 Level 5

11. Schug-Pass C, Jacob DA, Rittinghausen J, Lippert H, Köckerling F (2012) Biomechanical properties of (semi-) synthetic glue for mesh fixation in endoscopic inguinal hernia repair. Hernia. doi:10.1007/s10029-012-1000-8 Level 5

12. Schug-Pass C, Lippert H, Köckerling F (2009) Fixation of mesh to the peritoneum using a fibrin glue: investigations with a biomechanical model and an experimental laparoscopic porcine model. Surg Endosc 23:2809-2815 Level 5

13. Rieder E, Stoiber M, Scheikl V, Poglitsch M, Dal Borgo A, Prager G, Schima H (2011) Mesh fixation in laparoscopic incisional hernia repair: glue fixation provides attachment strength similar to absorbable tacks but differs substantially in different meshes. J Am Coll Surg 212(1):80-86 Level 5

14. Penttinen R, Grönroos JM (2008) Mesh repair of common abdominal hernias: a review on experimental and clinical studies. Hernia 12:337-344 Level 5

15. Junge K, Rosch R, Klinge U, Saklak M, Klosterhalfen B, Peiper C, Schumpelick V (2005) Titanium coating of a polypropylene mesh for hernia repair: effect on biocompatibility. Hernia 9:115-119 Level 5

16. Pereira-Lucena CG, Artigiani-Neto A, Lopes-Filho GJ, Frazao CVG, Goldenberg A, Matos D, Linhares MM (2010) Experimental study comparing meshes made of polypropylene, polypropylene + polyglactin and polypropylene + titanium: inflammatory cytokines, histological changes and morphometric analysis of collagen. Hernia 14:299-304 Level 5

17. Burger JWA, Halm JA, Wijsmuller AR, ten Raa S, Jeekel J (2006) Evaluation of new prosthetic meshes for ventral hernia repair. Surg Endosc 20:1320-1325 Level 5

18. Chatzimavroudis G, Koutelidakis I, Papaziogas B, Tsaganos T, Koutoukas P, Giamarellos-Bourboulis E, Atmatzidis S,
Atmatzidis K (2008) The effect of the type of intraperitoneally implanted prosthetic mesh on the systemic inflammatory response. Hernia 12:277-283 Level 5

19. Schreinemacher MHF, Emans PJ, Gijbels MJJ, Greve JWM, Beets GL, Bouvy ND (2009) Degradation of mesh coatings and intraperitoneal adhesion formation in an experimental model. Br J Surg 96:305-313 Level 5

20. Ott R, Hartwig T, Tannapfel A, Blatz R, Rodloff AC, MadajSterba P, Möbius C, Köckerling F (2007) Biocompatibility of bacterial contaminated prosthetic meshes and porcine dermal collagen used to repair abdominal wall defects. Langenbecks Arch Surg 392:473-478 Level 5

21. Fortelny RH, Pettert-Puchner AH, Walder N, Mittermayr R, Öhlinger W, Heinze A, Redl H (2007) Cyanoacrylate tissue sealant impairs tissue integration of macroporous mesh in experimental hernia repair. Surg Endosc 21:1781-1785 Level 5

22. Scheidbach H, Tamme C, Tannapfel A, Lippert H, Köckerling F (2004) In vivo studies comparing the biocompatibility of various polypropylene meshes and their handling properties during endoscopic total extraperitoneal (TEP) patch plasty. Surg Endosc 18:211-220 Level 5

23. Schug-Pass C, Tamme C, Tannapfel A, Köckerling F (2006) A lightweight polypropylene mesh (TiMesh) for laparoscopic intraperitoneal repair of abdominal wall hernias. Surg Endosc 20:402-409 Level 5

24. Schug-Pass C, Sommerer F, Tannapfel A, Lippert H, Köckerling F (2008) Does the additional application of a polylactide film (SurgiWrap) to a lightweight mesh (TiMesh) reduce adhesions after laparoscopic intraperitoneal implantation procedures? Experimental results obtained with the laparoscopic porcine model. Surg Endosc 22:2433-2439 Level 5

25. Schulz KF, Altman DG, Moher D (2010) CONSORT 2010 statement: updated guidelines for reporting parallel group randomized trials. Ann Intern Med 152:726-732

26. Koch A, Bringman S, Myrelid P, Smeds S, Kald A (2008) Randomized clinical trial of groin hernia repair with titaniumcoated light-weight mesh compared with standard polypropylene mesh. Br J Surg 95:1226-1231 Level 1 B

27. Schopf S, von Ahnen T, von Ahnen M, Schardey H (2011) Chronic pain after laparoscopic transabdominal preperitoneal hernia repair: a randomized comparison of light and extralight titanized polypropylene mesh. World Surg 35:302-310 Level 1 B

28. Bittner R, Schmedt CG, Leibl BJ, Schwarz J (2011) Early Postoperative and One Year Results of a Randomized Controlled Trial Comparing the Impact of Extralight Titanized Polypropylene Mesh and Traditional Heavyweight Polypropylene Mesh on Pain and Seroma Production in Laparoscopic Hernia Repair (TAPP). World J Surg 35:1791-1797 Level 1 B

29. Fortelny RH, Petter-Puchner AH, May C, Jaksch W, Benesch T, Khakpour Z, Redl H, Glaser KS (2012) The impact of a traumatic fibrin sealant vs. staple mesh fixation in TAPP hernia repair on chronic pain and quality of life: results of a randomized controlled study. Surg Endosc 26:249-254 Level 1 B

30. Peeters E, Spiessens C, Oyen R, De Wever L, Vanderschueren D, Pennickx F, Miserez M (2010) Laparoscopic inguinal hernia repair in men with lightweight meshes may significantly impair sperm motility. Ann Surg 252(2):240-246 Level 1 B

31. Peeters E, Spiessens C, Oyen R, De Wever L, Vanderschueren D, Pennickx F, Miserez M (2012) Sperm motility after laparoscopic inguinal hernia repair with lightweight meshes: 3-year follow-up of a randomised clinical trial. Hernia. doi:10.1007/s10029-0121028-9 Level 1 B

32. Horstmann R, Hellwig M, Classen C, Röttgermann S, Palmes D (2006) Impact of polypropylene amount on functional outcome and quality of life after inguinal hernia repair by the TAPP 
procedure using pure, mixed, and tianium-coated meshes. World J Surg 30:1742-1749 Level 2 B

33. Fortelny RH, Schwab R, Glaser KS, Puchner KU, May C, König

F, Redl H, Petter-Puchner AH (2008) The assessment of quality of life in a trial on lightweight mesh fixation with fibrin sealant in transabdominal preperitoneal hernia repair. Hernia 12:499-505 Level 4
34. Moreno-Agea A, Carrillo-Alcaraz A, Sora-Aledo V (2012) Randomized clinical trial of laparoscopic hernia repair comparing titanium-coated lightweight mesh and medium-weight composite mesh. Surg Endosc doi: 10.1007/s00464-012-2425-y (Level 1 B) 